\title{
Artifacts from the Electric Field build up in the Microbeam Analysis of Insulating Materials
}

O. Jbara, M. Belhaj and S. Fakhfakh

\section{LASSI/DTI UMR CNRS 6107, Faculté des Sciences BP 1039, 51687 REIMS CDX 2 France}

When certain insulating materials are subjected to electron irradiation as in Scanning Electron Microscopy (SEM) or associated analytical techniques such as Electron Probe Microanalysis (EPMA), without prior metallic coating, charging phenomena occur. The build up electric field in the vacuum resulting from the trapped electrons in the sample leads to an electronic source irradiating back the sample as well as the sample holder. This source is caused by the secondary (SEs) and backscattered (BSEs) electrons emitted from the sample which are first accelerated and collimated on the poles pieces by the external electric field and next backscattered (Fig.1) . The consequences concern, an anomalous contrast in the secondary electron image and a distortion of the experimentally observed x-ray bremsstrahlung spectrum. The anomalous contrast is due to the so-called pseudo mirror (PM) effect [1] and the image obtained (Fig.2a) contains two contrast contributions corresponding not only to a distorted view of the SEM chambre (as for mirror image [2]) but also to a view of the sample scan area. Unlike the mirror image (Fig.2b) the PM effect leads to less resolved details to such a point (especially for flat samples) that they are often assigned to a local change of SE emission. The explanation of the pseudo mirror described elsewhere [1] is based on the distortion of the angular distribution of secondary electrons, which is more elongated in the direction of the external electric field and also on modification of their spectral distribution that is shifted to higher energies (the results of measurements of this distribution that have been carried out using a special spectrometer [3] are published elsewhere [4]). Returning to the distorted x-ray spectrum, the bremsstrahlung is the sum of two processes different in origin (see fig 3): the excitation of the bremsstrahlung due to the primary electron beam and that due to the additional source formed by the BSEs emitted from the pole pieces. As a result, the position of the measured high energy cut-off limit of bremsstrahlung radiation detected from the charged sample is not consistent with the effective landing energy of the primary electrons and hence its position is not directly connected with the surface potential of charged sample [5]. In fact, the surface potential deduced from the distorted spectrum is $-2.3 \mathrm{kV}$ while that measured using an electron spectrometer is $-11.5 \mathrm{kV}$ [4]. It can be also seen, the presence of $\mathrm{CrK} \alpha$ and $\mathrm{NiK} \alpha$ lines (issued from the sample holder irradiated by the delocalised additional source) even though the primary beam irradiated area is free from the corresponding elements (see spectrum 2 in fig.3). From the EPMA point of view, operating with sufficiently high primary energies, hence creating x-ray from the chambre walls by accelerated SEs, constitutes an impediment to elemental identification of electron-irradiated insulators especially if the sample and the chamber contain the same elements.

\section{References}

[1] M. Belhaj et al, Scanning, 22 (2000), 352-356.

[2] B. Vallayer, G. Blaise and D. Treheux, Rev. Scient. Inst, 70, 3102 (1999).

[3] E.I Rau and V.N.E. Robinson, Scanning 18, 556-561 (1996).

[4] O. Jbara et al, Rev. Sci. Instr., 72 (2001), 1788-1795.

[5] M. Belhaj et al, Appl. Surf. Sci., 177 (2001), 58-65. 


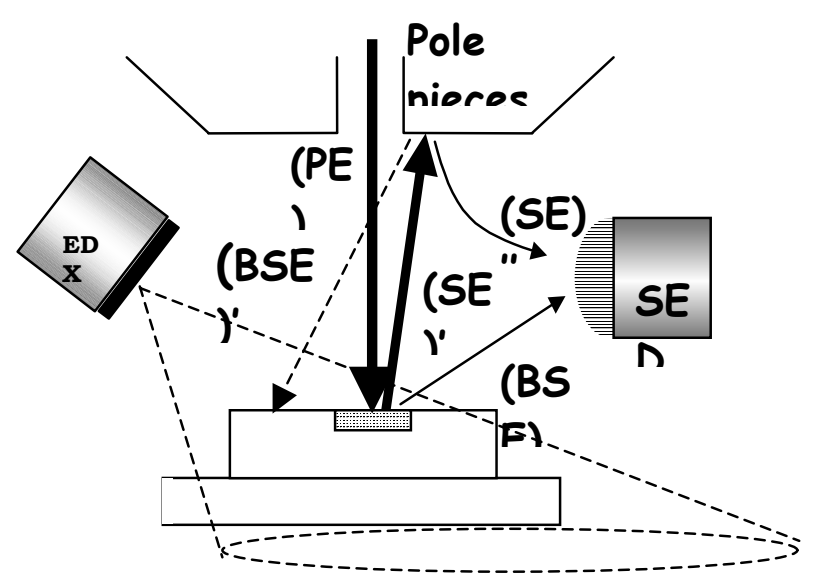

Fig.1

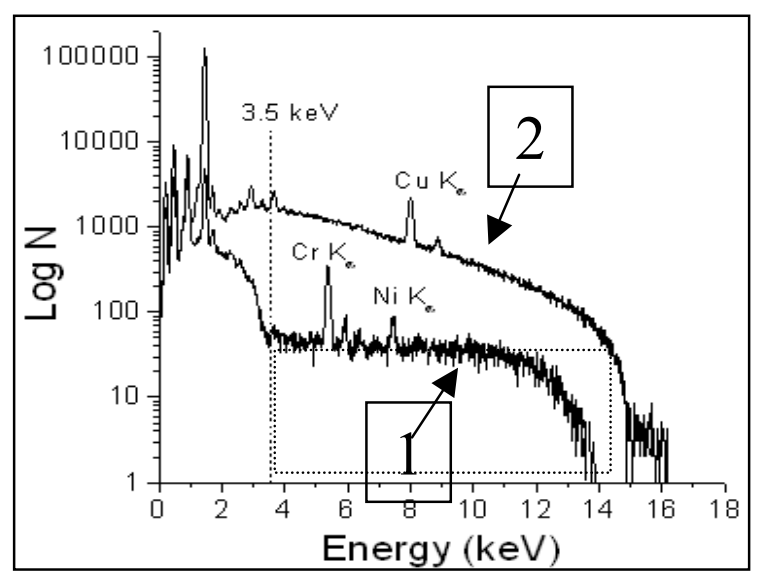

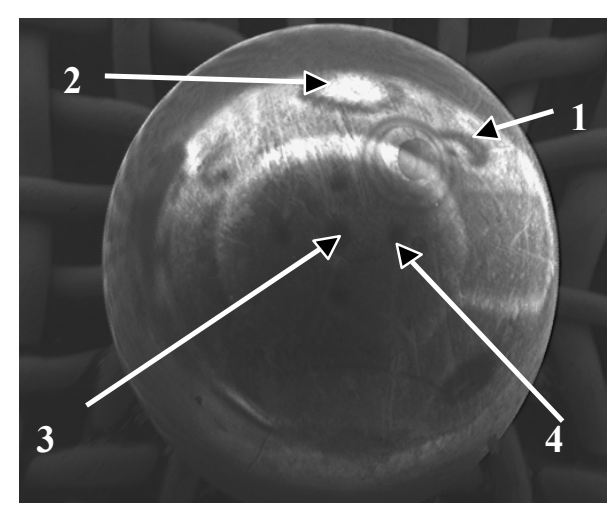

Fig.2a

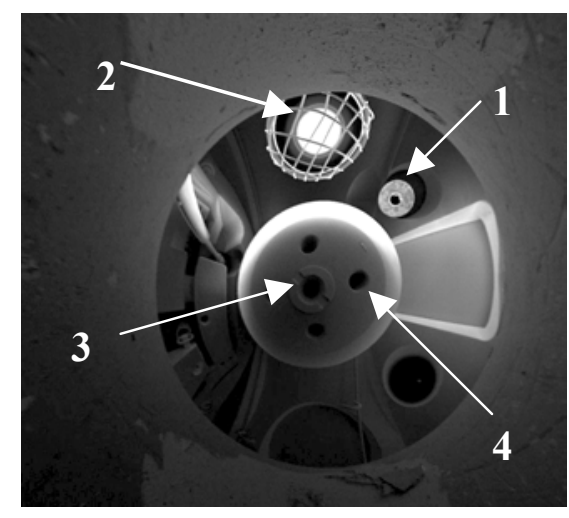

Fig.2b

\section{Fig.3}

Fig. 1 Schematic drawing of the mechanism formation of the pseudo mirror image. The scanning of the SEM inner shell by the relatively high energy SE pseudo beam (SE) is in synchronism with the scanning of the surface sample by the primary electron beam (PE).

Fig. 2 (a): Mirror image of the scanning electron microscope (SEM) chamber obtained at $10 \mathrm{kV}$ primary accelerating voltage. The charging was performed at $20 \mathrm{kV}$ on . Details of the SEM inner shell are clearly distinguished. (1) collimator of the SiLi detector (2) secondary electron detector (grid and scintillator) (3) output electron gun aperture (4) screw holes.

(b): Pseudo mirror image recorded at $20 \mathrm{kV}$ primary beam accelerating voltage. Horizontal width $=1.6 \mathrm{~mm}$. The insulating sample was a $\mathrm{Al}_{2} \mathrm{O}_{3}$-sapphire sphere.

Fig. 3 (1) X-ray spectra (in logarithmic scale) recorded at $16 \mathrm{keV}$ primary beam energy and $10 \mathrm{nA}$ primary beam current: (1) from the non-grounded $\mathrm{Cu}$-coated $\mathrm{Al}_{2} \mathrm{O}_{3}$; (2) :from the grounded $\mathrm{Cu}$-coated $\mathrm{Al}_{2} \mathrm{O}_{3}$. The time acquisition was $100 \mathrm{~s}$. 